\title{
Quand l'étude génétique est contemporaine du processus de création : nouveaux objets, nouveaux problèmes
}

\section{Nicolas Donin}

\section{(2) OpenEdition}

\section{Journals}

Édition électronique

URL : http://journals.openedition.org/genesis/327

DOI : 10.4000/genesis.327

ISSN : 2268-1590

Éditeur :

Presses universitaires de Paris Sorbonne (PUPS), Société internationale de génétique artistique littéraire et scientifique (SIGALES)

Édition imprimée

Date de publication : 10 octobre 2010

Pagination : 13-36

ISBN : 978-2-84050-711-6

ISSN : 1167-5101

\section{Référence électronique}

Nicolas Donin, «Quand l'étude génétique est contemporaine du processus de création : nouveaux objets, nouveaux problèmes », Genesis [En ligne], 31 | 2010, mis en ligne le 20 septembre 2012, consulté le 20 avril 2019. URL : http://journals.openedition.org/genesis/327 ; DOI : 10.4000/ genesis.327 


\title{
Quand l'étude génétique est contemporaine du processus de création : nouveaux objets, nouveaux problèmes
}

\author{
Nicolas Donin
}

$\mathrm{L}$ A SAISIE DU PROCESSUS CRÉATEUR dans le temps même de son effectuation, bien avant d'être une problématique génétique latente ou émergente, a eu une longue existence qu'il serait difficile de passer sous silence, étant donné son importance dans l'imaginaire collectif : celle d'un fantasme récurrent de l'amateur d'art du $\mathrm{Xx}^{\mathrm{e}}$ siècle. Une formulation canonique entre toutes en est donnée dans le prologue du Mystère Picasso (1955) de Clouzot. Tandis que la caméra accompagne l'artiste dans la pénombre de l'atelier épuré où le drame va se dérouler, une voix off (celle du cinéaste) énonce la théorie de ce documentaire :

On donnerait cher pour savoir ce qui s'est passé dans la tête de Rimbaud pendant qu'il écrivait le Bateau ivre, dans la tête de Mozart pendant qu'il composait la Symphonie Jupiter, pour connaître ce mécanisme secret qui guide le créateur dans son aventure périlleuse. Grâce à Dieu, ce qui est impossible pour la poésie et la musique est réalisable en peinture. Pour savoir ce qui se passe dans la tête d'un peintre, il suffit de suivre sa main. [Ici, on voit Picasso s'approcher d' un support vierge avec un feutre noir et commencer à dessiner.] Vous allez voir, c'est une drôle d'aventure que celle du peintre : il marche, il glisse en équilibre sur la corde raide : une courbe l'entraîne à droite, une tache l'entraîne à gauche. S'il rate son rétablissement, tout bascule, tout est perdu. Le peintre avance en tâtonnant comme un aveugle dans l'obscurité de la toile blanche. [...] Pour la première fois, ce drame quotidien et confidentiel de l'aveugle de génie va se jouer en public, puisque Pablo Picasso a accepté de le vivre aujourd'hui, devant vous, avec vous 1 .

Cette entrée en matière, en soi emphatique et datée, est même affligeante si on la met en regard de la sobre perfection du dispositif expérimental qu'elle est censée introduire, ainsi que de la finesse de construction de l'ensemble du long-métrage. Dans la première moitié du film, Picasso dessine aux feutres de couleur épais sur un papier translucide placé verticalement devant lui, la caméra se trouvant de l'autre côté et captant l'apparition du moindre trait - avec un effet de surprise permanent dû à l'impossibilité, depuis cet emplacement, de deviner où voltige la main de l'artiste en dehors de ses actes d'inscription. Pour la seconde moitié, il passe à la peinture de grand format : mettant en chantier une large toile (aux proportions cohérentes avec la pellicule) qu'il va retoucher sans cesse et recouvrir ainsi plusieurs fois, Picasso s'éclipse entre chaque bref moment de travail de telle sorte que la caméra, placée cette fois-ci face à la toile (tout comme lui), puisse enregistrer tous les états successifs de l'œuvre en cours. Le corps de l'artiste, devenu obstacle, s'efface devant l'œuvre ; et la machine cinématographique, ne pouvant plus capter le geste en train de se faire, se réduit bientôt à une sorte de chronophotographe.

1. Henri-Georges Clouzot, Le Mystère Picasso (1955), Arte vidéo, 2007, 0’06”-1'22”. On rêverait de lire/voir une étude génétique de ce film, à ma connaissance inexistante. Il en va de même à propos de la série de documentaires (injustement méconnue) sur la musique réalisée par Clouzot quelques années plus tard avec Herbert von Karajan, dans la droite ligne du Mystère Picasso.

De la fiction audiovisuelle... 
Si la majeure partie du film fonctionne admirablement sans commentaires (rares sont les intermèdes ou dialogues interrompant la musique d'accompagnement hispanisante composée par Georges Auric), ce n'est pas seulement grâce aux qualités de peintre (ainsi que d'acteur et de dramaturge) de Picasso. C'est aussi parce que différentes possibilités de montage cinématographique sont exploitées graduellement, créant un mouvement, voire une narration implicite - de l'homogénéité temporelle (lorsque le temps du film est le temps effectif du dessin) aux effets d'accélération/décélération, en passant par la lecture d'une séquence dans le sens inverse, qui donne au spectateur l'impression de « détricoter » la structure d'un dessin dont il venait d'assimiler, pas à pas, tout le cheminement constructif. L'expérience tient la route, même si ses présupposés officiels sont douteux. En documentant de façon rigoureuse et, somme toute, modeste le «drame quotidien et confidentiel » initialement annoncé, le film de Clouzot s'avère solidaire (quoique à son insu) d'enjeux théoriques autres, portés par le dispositif mis au point mais non explicités, qui pourraient être, par exemple, ceux de la «science » rêvée par Picasso lui-même une dizaine d'années plus tôt :

Sans doute existera-t-il un jour une science, que l'on appellera peut-être « la science de l'homme », qui cherchera à pénétrer plus avant l'homme à travers l'homme-créateur... Je pense souvent à cette science et je tiens à laisser à la postérité une documentation aussi complète que possible... Voilà pourquoi je date tout ce que je fais ${ }^{2}$..

L'idéal scientifique suggéré par ce propos rapporté par Brassaï n'a guère progressé du vivant du peintre (en dépit des prétentions de la psychologie de la créativité, contemporaines de la collaboration entre Clouzot et Picasso). En revanche, le fantasme de filmer la création dans le cerveau du créateur est loin d'avoir disparu - y compris parmi les chercheurs. Pour s'en tenir à des exemples pris dans le champ musical, y a-t-il une si grande distance entre les présupposés de l'une des très rares études récentes en psychologie cognitive de la composition espérant « plonger dans l'esprit du compositeur ${ }^{3}$ », et la fiction audiovisuelle prophétique rêvée par Bernard Gavoty, plusieurs années avant le Mystère Picasso, au cours de son grand entretien avec Arthur Honegger?

Combien il serait précieux, cependant, de réaliser une sorte de film qui enregistrerait pour la postérité les phases principales de la conception et de la réalisation d'un chef-d'œuvre musical : une sorte de reportage qui exigerait, pour être parfait, que la caméra fût dans le cerveau et non pas seulement dans le bureau du compositeur ${ }^{4}$.

... au projet scientifique
Si la critique génétique commence, assurément, là où s'arrête la mystique du « mécanisme secret » de l'artiste inspiré, échappe-t-elle pour autant au genre d'apories que l'on vient d'évoquer? L'affranchissement originaire de la discipline à l'égard des discours hypostasiants (la création serait dans le cerveau, dans l'inconscient, dans la société, etc.) ne s'est pas affirmé

2. Brassaï, Conversations avec Picasso, Paris, Gallimard, 1964, p. 123 (note du lundi 6 décembre 1943).

3. Stephen McAdams, « Problem-Solving Strategies in Music Composition: A Case Study », Music Perception, vol. XXI, $\mathrm{n}^{\circ} 3,2004$, p. 391 ( The problem is to delve into the mind of a composer in the process of composing »). Jugée impraticable, cette ruée dans le cerveau est rapidement écartée au profit - faute de mieux - d'un recueil a posteriori de données et de propos du compositeur.

4. Arthur Honegger, Je suis compositeur, Paris, Conquistador, 1951, p. 89-90 (en italique dans le texte). 
sans dangers : à force de ne se laisser annexer ni par l'histoire, ni par la linguistique, ni par la sémiologie, ni par l'anthropologie, ni par la philologie - comme des représentants de ces disciplines ont pu en faire le vœu à telle ou telle occasion -, la génétique a dissipé des quiproquos et consolidé son indépendance, mais elle a aussi vu s'amenuiser les occasions de confronter durablement ses objets et ses frontières avec ceux des nombreuses disciplines sœurs qui l'entourent - au risque de figer sa propre identité. Toutes choses égales par ailleurs, la musicologie a couru des risques comparables (aggravés en l'occurrence par une profonde inconscience théorique) durant la majeure partie de son long siècle d'existence 5 . Si les travaux sur la composition musicale présentés ici même ont été délibérément élaborés à l'intersection de plusieurs disciplines non musicologiques (à commencer par la critique génétique), c'est précisément parce que la musicologie avait paru échouer, seule, à constituer la composition en objet d'étude à part entière. Peut-être l'exposé de cette dynamique de recherche toujours en cours pourra-t-il contribuer à la réflexion sur l'interdisciplinarité nécessaire au projet d'une génétique générale, capable d'englober toutes les formes de genèse artistiques. Au minimum, il vise en tout cas à recenser quelques-uns des problèmes spécifiques posés par l'étude d'œuvres contemporaines à proximité de leurs circonstances de réalisation, voire en même temps que cette dernière.

Le Mystère Picasso, avec ses clichés grand public comme avec ses séquences intelligentes et problématiques, ne posait pas que des questions génétiquement triviales. Y crevaient l'écran tout à la fois l'immense intérêt d'une génétique en quelque sorte inchoative (qui saisirait l'œuvre en train de se faire) et l'impossibilité de concrétiser cette dernière avec la même latitude, avec les mêmes hypothèses et procédures que celles dont dispose le chercheur face à un dossier génétique plus traditionnel, constitué d'un ensemble fini de traces inanimées de l'activité créatrice.

Un appel à la génétique « in vivo » a été formulé par Pierre-Marc de Biasi dès 1993 dans la section conclusive de son texte sur l' « horizon génétique », pointant l'élargissement souhaité d'une discipline indexée exclusivement, en ses débuts, sur la production verbale6. L'auteur suggérait d' " imaginer un nouveau type de solidarité entre la création et la recherche » dans lequel « des artistes, des scientifiques et des penseurs contemporains [...] concernés par la critique génétique » accepteraient de donner aux chercheurs un accès direct à leur travail :

[...] le chercheur peut aujourd'hui envisager d'étudier la création contemporaine, en allant observer et saisir son objet en temps réel dans l'espace même de sa genèse : auprès du metteur en scène qui tourne son film, dans le laboratoire de biologie ou d'astrophysique où travaillent les chercheurs, dans le cabinet d'architecte où s'élabore un projet d'immeuble? .

Certains avantages et défauts de cette « recherche génétique sur le terrain 8 » étaient pointés : en permettant non seulement la « collecte à la source » mais aussi l' « observation directe » et

5. Voir Revue d' histoire des sciences humaines, $\mathrm{n}^{\circ}$ 14, « Musique et sciences humaines, un rendez-vous manqué ? », 2006.

6. Pierre-Marc de Biasi, «L'horizon génétique », dans Les Manuscrits des écrivains, Paris, CNRS Éditions/Hachette, 1993, p. 238-259 - l'expression « in vivo » se trouve p. 258.

7. Pierre-Marc de Biasi, ibid., p. 258.

8. Ibid., p. 259. 
« la possibilité d'interviewer [1']auteur ${ }^{9}$ », elle garantirait une complétude et une précision du dossier génétique sans précédent; en même temps, cela inverserait les habitudes de travail du généticien, confronté non plus à des traces éparses au statut équivoque, mais à un trop-plein d'informations où l'essentiel se trouverait nécessairement noyé dans l'accessoire ; enfin, « la présence explicite ou implicite d'un observateur qui entend tout examiner modifie[rait] le climat de la genèse », ce dont « l'analyse [devrait] tenir compte10 ». Nous pourrions ajouter à cette liste le danger de se tromper de lieu d'observation : au fond, pourquoi l'essentiel de l'activité créatrice et de ses traces devraient-ils se trouver sur le plateau du tournage (pour le metteur en scène) ou sur la paillasse (pour le biologiste) ? La tentation du déterminisme des lieux, l'hypothèse d'un accès immédiat à l'acte créateur grâce à la présence physique du chercheur, risqueraient en effet de minorer une conséquence importante de la démarche observationnelle : lorsqu'on s'approche non seulement d'un « processus » mais de l'activité qui détermine ce dernier, le créateur n'apparaît pas seulement comme l'opérateur d'une méthode inédite à déchiffrer, mais comme un individu engagé dans de multiples tâches (conscientes et inconscientes) au sein d'une fragile économie cognitive au sein de laquelle il peut - ou pas - accepter la présence insistante du généticien. C'est aussi cette économie cognitive que ce dernier devra démêler pour y retrouver ses objets.

Qu'il s'agisse du réglage de l'interaction entre observateur(s) et observé(s), ou du problème de l'hypermnésie (notamment numérique) qui risque de transformer le généticien en archiviste, les questions étaient posées par de Biasi en termes de difficultés pratiques à anticiper. Les traiter plutôt en termes épistémologiques aurait sans doute conduit à nommer de nouveaux partenaires (ou adversaires) de la discipline : l'ethnologie et la sociologie, en plus de la psychologie qui, sur d'autres thématiques, est depuis longtemps identifiée. À l'époque de ce texte, cependant, rien n'avait été tenté qui justifiât d'entrer dans un tel débat. En va-t-il autrement aujourd'hui ? Pour l'heure, bien peu d'études de cas ont été réalisées et la notion d'une génétique in vivo ${ }^{11}$ serait restée à l'état de potentialité sans le récent travail d'Irène Fenoglio sur un conte de Pascal Quignard ${ }^{12}$. L'écrivain communique à la chercheuse, entre juin 2003 et août 2004, par envoi postal ou de la main à la main, plusieurs versions du conte successivement intitulé Bernon l' enfant puis Fête des Chants du Marais ${ }^{13}$. Ce sont des tirages papier, généralement annotés à la main, et datés - permettant dans la constitution du dossier génétique « une systématicité qui entraîne un classement chronologique élémentaire et sûr14 ». Le but de l'étude est de " donner à voir la façon dont l'un des plus grands de nos écrivains contemporains [...] utilise les possibilités qu'offre le traitement de texte par ordinateur pour écrire 15 ». La principale caractéristique mise en évidence dans l'article issu de cette étude est l'importance des relectures, que Fenoglio corrèle au traitement de texte

\footnotetext{
9. Ibid., p. 258

10. Ibid., p. 259.

11. Ibid., p. 258

12. Irène Fenoglio, «Fête des Chants du Marais, un conte inédit de Pascal Quignard. Genèse in vivo et "traitement de texte" ", Genesis, n 27, 2006, p. 73-93.

13. La version définitive (?) apparaît, quant à elle, sous le titre La Fête des chants du marais, dans La Barque silencieuse, Paris, Grasset, 2009.

14. Irène Fenoglio, «Fête des Chants du Marais, un conte inédit de Pascal Quignard », art. cit., p. 81.

15. Ibid., p. 73
} 
informatique. Ce dernier, écrit-elle, « offre un support de corrections possibles renouvelables à l'infini, mais dans le même temps il sollicite la relecture qui peut toujours se faire au clair d'une page "propre" et donc, aussi, à nouveau, d'éventuelles corrections 16 ». Cette hypothèse, fortement dépendante du type de source exploité, est contresignée par l'écrivain :

Pour ce qui est de Pascal Quignard, cela m'a été confirmé par l'auteur : depuis qu'il utilise l'ordinateur, il travaille beaucoup plus ses textes. La machine à écrire obligeant à « retaper » l'ensemble d'un texte lui imposait des limites ${ }^{17}$.

Transgressant un habitus disciplinaire dont elle fait apparaître le caractère inutilement contraignant, cette étude n'a cependant pas entraîné un positionnement de la critique génétique par rapport à certaines des questions cruciales soulevées par ce nouveau type d'objet : quel accès à l'atelier est possible et souhaitable ? Comment régler l'interaction avec un artiste dont on souhaite prendre le processus créateur pour objet ? Comment définir initialement l'objet de l'étude, puisque cet objet est alors largement inconnu et qu'il n'est pas cadré par une raison archivistique ? Dès lors que la génétique est in vivo, peut-on et doit-on analyser le devenir-œuvre sans considérer en même temps l'activité ? Autant de questions auxquelles ont dû également s'affronter les études menées, au cours de la décennie passée, par l'équipe Analyse des pratiques musicales (Ircam-CNRS) 18 en collaboration avec un (puis plusieurs) compositeur(s), qu'il s'agit ici d'introduire. Les pages qui suivent présentent les principes méthodologiques mis en œuvre dans plusieurs de ces travaux, sans les illustrer par des exemples de résultats précis (dont la technicité musicale et la longueur excéderaient le cadre de cet article), mais en indiquant en notes de bas de page une sélection de références bibliographiques documentant chaque projet.

Un mot tout d'abord sur le programme de recherche dans le cadre duquel ces travaux ont été engagés. Associant des problématiques musicologiques et des méthodes issues des sciences humaines dans leur diversité (de l'histoire à l'anthropologie cognitive en passant par... la critique génétique), il vise à mieux comprendre les pratiques, œuvres et savoir faire spécifiques de la «musique contemporaine ${ }^{19}$ »- pour laquelle l'Ircam constitue à la fois un creuset historique et un lieu d'observation particulièrement riche. Du fait de sa double inscription, d'une part dans la musicologie (qui privilégie traditionnellement l'étude des partitions et de leurs auteurs), d'autre part dans une institution dédiée à la création (l'Ircam héberge des studios informatisés, une saison de concerts et un cursus pédagogique de composition assistée par ordinateur), les pratiques de composition actuelles constituent naturellement l'objet central de ce programme de recherche.

16. Ibid., p. 80 .

17. Ibid.

18. Une présentation générale de l'équipe et un ensemble de ressources documentaires et bibliographiques sont régulièrement mis à jour à l'adresse <http://apm.ircam.fr/>.

19. Puisque ce syntagme ne cesse de se détacher de ce qui était son signifiant usuel dans le dernier tiers du siècle dernier, il convient d'être explicite : par «musique contemporaine » nous entendrons ici la création musicale contemporaine de tradition occidentale savante, généralement indexée sur l'écriture de partitions et destinée à l'exécution dans des salles de concert classique et d'opéra.

\section{Enrôler le créateur dans la démarche génétique?}


Une première étude, qui s'est avérée déterminante, a été menée à partir de 2003 sur et avec le compositeur français Philippe Leroux (né en 1959). Elle consistait en une reconstruction du processus de composition de Voi(rex), une œuvre pour voix, petit ensemble instrumental et parties électroniques composée en 2002 (avec la participation d'un réalisateur en informatique musicale, Frédéric Voisin, pour l'électronique élaborée à l'Ircam) et donnée en création en janvier 2003. Pendant la composition, plus régulièrement qu'à son habitude, Leroux avait porté des annotations ponctuelles dans la marge inférieure de sa partition manuscrite, par exemple : «voix : Taléa (analyse rythmique poème Donatienne) », « Montréal », « ré\# note principale du IIMvt ${ }^{20}$. Ces inscriptions dénotaient de façon elliptique des opérations de composition importantes et/ou des identifiants (numériques, solfégiques, verbaux) susceptibles de faciliter la remémoration ultérieure du travail accompli, que ce soit à l'occasion d'autres projets d'œuvres nécessitant le recyclage de matériaux ou techniques de Voi(rex), ou bien encore de situations pédagogiques (cours de composition). Par ailleurs, Leroux avait conservé de nombreux documents de travail - esquisses, brouillons et autres notes, formant les ingrédients d'un riche dossier génétique (voir fig. 1 à 3). Il était dès lors envisageable d'articuler étroitement ce qui aurait pu aussi bien constituer une série de recherches distinctes et parallèles : une analyse musicale de la partition, une analyse génétique de l'œuvre (ou plus précisément de sa rédaction, distinguée de sa phase préparatoire moins précisément documentable), et une analyse cognitive du travail créateur (basée sur les indications que le compositeur pourrait fournir, entre autres, au moyen des aide-mémoire par lui confectionnés à la façon du Petit Poucet). Cette articulation a été effective parce que les arrière-plans disciplinaires des chercheurs (Jacques Theureau 21 et moi-même) se sont agencés au sein d'un cadre méthodologique commun, et bien sûr parce que la nature des données ainsi que l'aimable participation du compositeur la rendaient possible.

20. Sélection d'inscriptions relatives au premier mouvement de Voi(rex), à l'aplomb respectivement des mesures 21-22 (manuscrit autographe de la partition, p. 4), 55 (p. 7), 67 (p. 14).

21. Chercheur au CNRS (aujourd'hui retraité), principal introducteur de l'anthropologie cognitive dans sa discipline d'origine, l'ergonomie, Jacques Theureau a cofondé en 2003 l'équipe Analyse des pratiques musicales, où il a conçu et mené plusieurs travaux de recherche sur les activités musicales contemporaines en collaboration avec Samuel Goldszmidt et l'auteur de ces lignes (voir <http://apm.ircam.fr/membre/JT/>). 


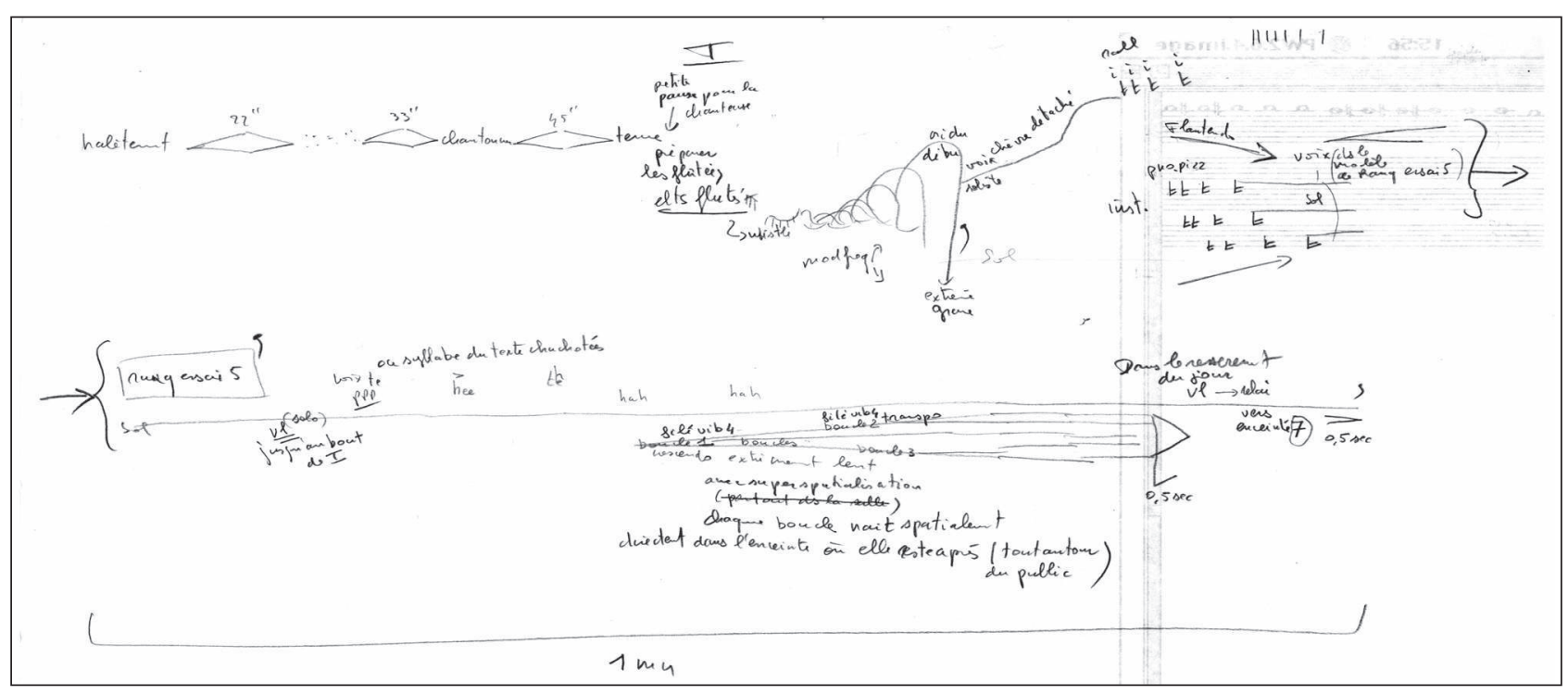

Fig. 1 : Philippe Leroux, brouillon séquentiel du mouvement I de Voi(rex) (coll. de l'auteur)

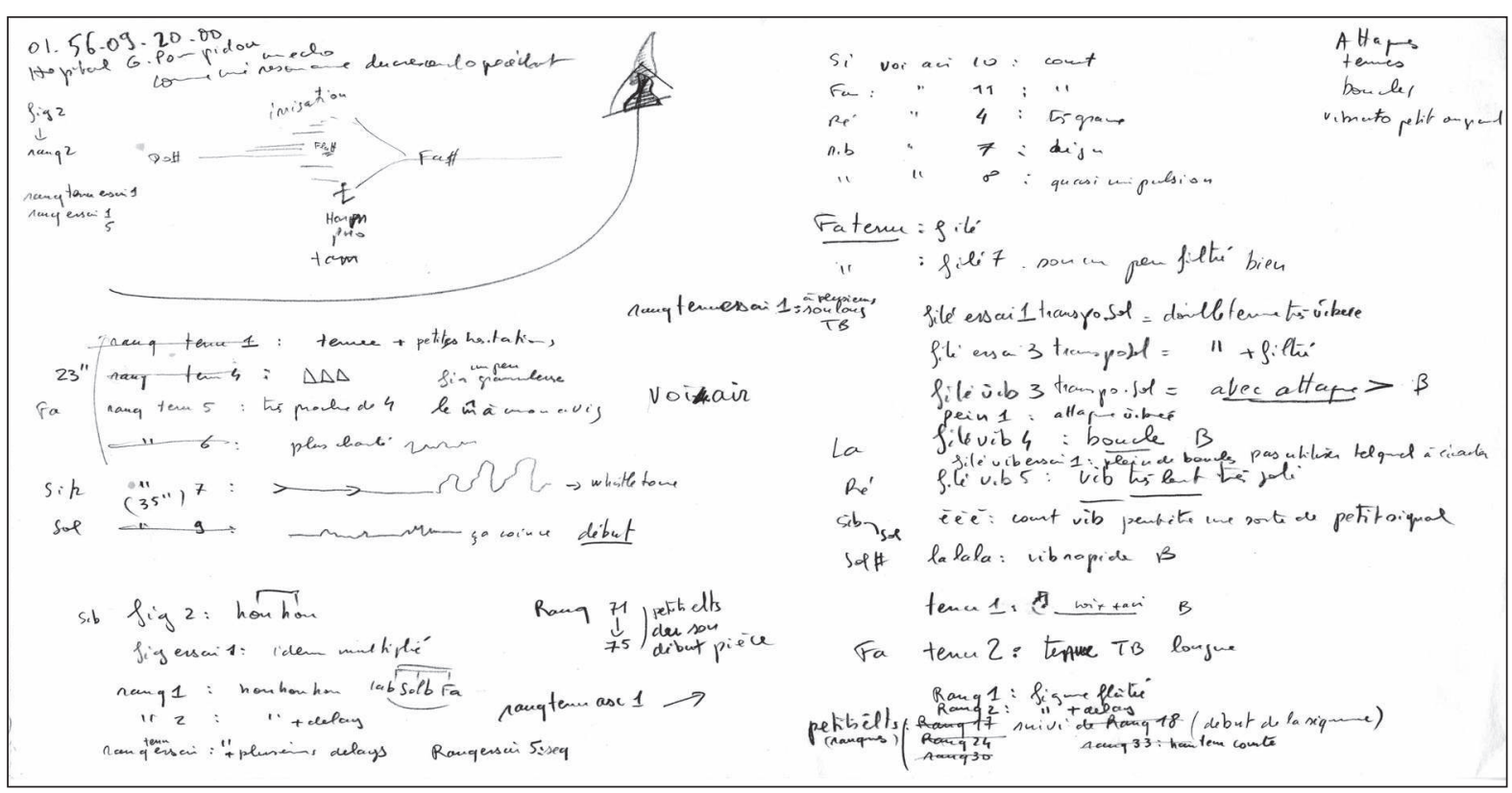

Fig. 2 : Philippe Leroux, brouillon du mouvement I de Voi(rex) contenant principalement des descriptions de différents sons enregistrés lors d'une séance de travail préparatoire avec la chanteuse, et sélectionnés pour ce mouvement (coll. de l'auteur) 


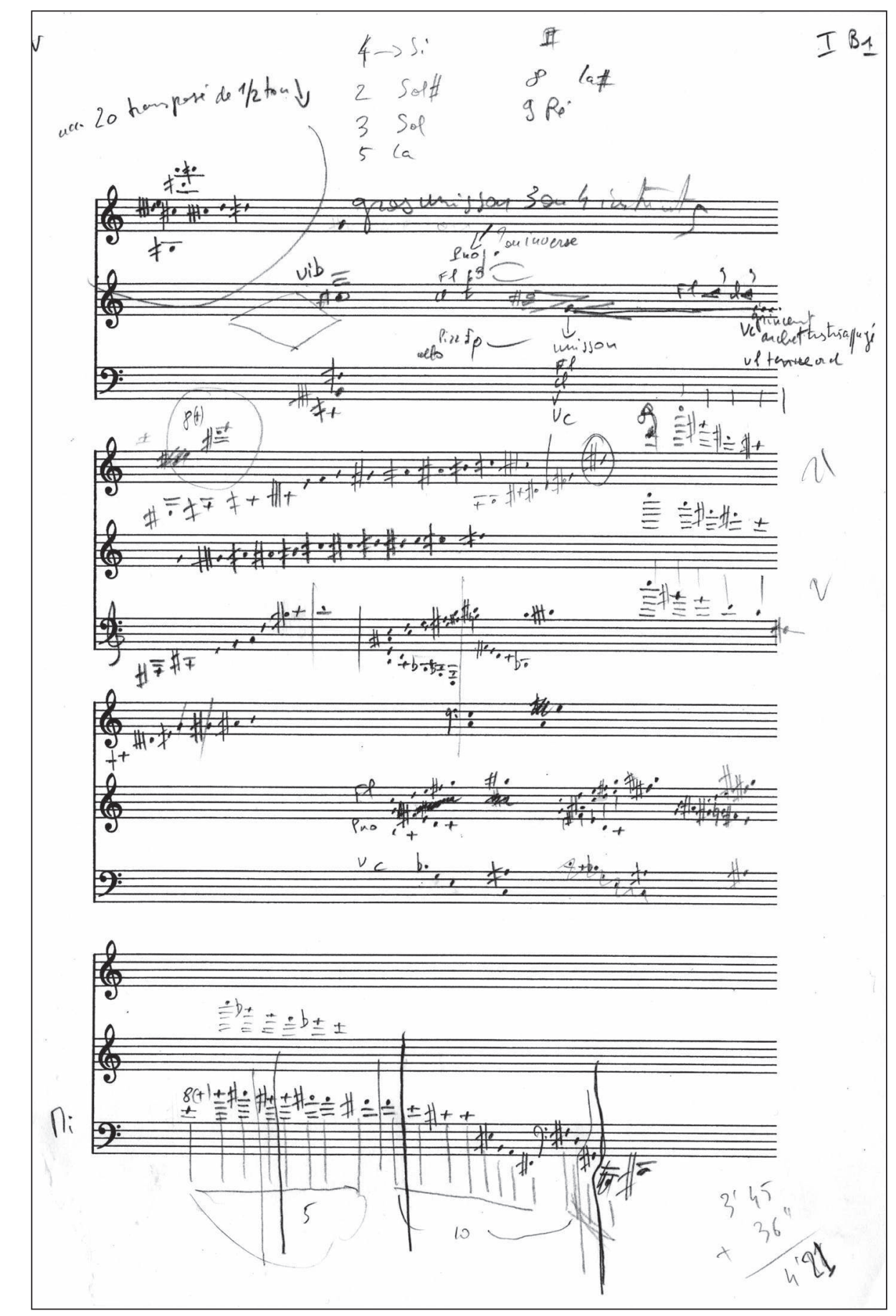

Fig. 3 : «Esquisse» (pendant la rédaction) de plusieurs passages du premier mouvement de Voi(rex) : détermination des hauteurs et de l'instrumentation

de passages préfigurés dans des brouillons tels que la figure 1 (coll. de l'auteur)

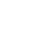


À l'exploration solitaire des traces de la genèse par les chercheurs sur la base d'un photocopiage intégral du riche ensemble de documents communiqué par le compositeur, s'est donc ajoutée une exploration collective, avec lui, de ces mêmes documents. Le dispositif de recueil de données consistait - après une séance préalable de familiarisation collective avec les documents de genèse et leur classement indigène, puis quelques essais méthodologiques - en une série d'une douzaine de séances de travail (en général, deux séances de deux à trois heures pour chacun des cinq mouvements de l'œuvre) suivant des règles élémentaires quant à leur déroulement et aux modalités d'interaction entre compositeur et chercheurs. Leur idée directrice était de reparcourir par la pratique (l'anglais dirait to reenact) le cours d'action du compositeur tout au long de la période de rédaction de la partition, en spécifiant les matériaux, outils et procédures requis dans chaque unité de composition de son œuvre (mouvement, section, portion de la partition écrite conjointement - ce qui peut aller d'une fraction de mesure à trois pages). Le choix du lieu tenait compte de la double nécessité, d'une part de disposer d'un environnement relativement comparable à celui avec lequel le compositeur avait interagi lorsqu'il élaborait l'œuvre, d'autre part d'éviter l'occupation de son atelier, ce dernier étant nécessairement configuré pour les projets actuellement engagés - du point de vue desquels Voi(rex) était devenu un produit fini, une ressource stabilisée, plutôt qu'un processus incertain à redécouvrir. La reconstitution se déroulait donc dans un lieu clairement distinct de l'atelier réel du compositeur, mais qui partageait avec ce dernier des caractéristiques importantes : calme et isolement relatifs, grande table de travail, possibilité d'installer un ordinateur portable et des haut-parleurs. Le produit des séances était conçu dès le départ comme une chose privée (un futur matériau de travail pour les phases d'analyse) et non pas publique (comme le sont les situations d'interlocution à propos de son travail auxquelles le compositeur est habitué, que ce soit avec des journalistes ou des musicologues). La parole du compositeur était sollicitée, non comme le substrat oral d'un art poétique, mais comme un moyen de verbaliser, au fur et à mesure de la consultation des traces de son activité passée, des aspects conscients ou semi-conscients de cette activité au moment de son accomplissement. Comment cet évitement des discours de représentation de soi était-il concrètement réalisé ?

Chaque séance comportait trois étapes successives. La première consistait, pour Philippe Leroux, à sélectionner dans le dossier génétique tous les éléments susceptibles de documenter la période d'écriture considérée. Cette dernière avait pour caractéristique remarquable de se laisser mécaniquement corréler à tout ou partie d'un mouvement de l'œuvre, sachant que la partition avait été rédigée page après page sur des feuillets doubles réunis en cahier, les esquisses les plus tardives étant effectuées au crayon à même le manuscrit sans retours en arrière. Au terme de cette première étape (généralement silencieuse), on disposait d'une reconstitution approximative de l'espace de composition propre au passage considéré : esquisses et brouillons (plus ou moins bien datés), ordinateur (contenant des programmes, des sons, etc.), portion de la partition manuscrite en cours de rédaction, éléments de la bibliothèque personnelle sollicités (livres et partitions de soi et d'autres compositeurs), etc. Mais tous ces documents étaient bien sûr dans leur état d'usage final, et non nécessairement dans l'état où ils se trouvaient à l'instant $t$ passé visé par la séance. La deuxième étape consistait alors en un commentaire de ce qui avait été sélectionné lors de la phase précédente : Leroux qualifiait plus ou moins précisément chaque 
document et il s'efforçait, pour les plus denses, de distinguer quelles inscriptions étaient nécessairement antérieures, contemporaines, postérieures à la période d'écriture considérée. Cette étape servait autant à engager la démarche de remémoration/verbalisation de la part du compositeur qu'à susciter des pistes d'interrogation pour la troisième (et principale) étape. Celle-ci consistait en une sollicitation conjointe du manuscrit autographe et des documents de travail qui avaient permis de mener à bien sa rédaction, unité de composition après unité de composition : le compositeur commentait (verbalement mais aussi par des gestes, des chantonnements, des mimiques) son activité passée au fur et à mesure, et les chercheurs relançaient ce commentaire en cas d'imprécision ou d'incohérence entre le commentaire et les traces disponibles (ou entre différentes affirmations du compositeur). Aucune question ni relance ne portait sur la composition en général : toutes se référaient à des opérations précises et/ou à des propos tenus par le compositeur sur de telles opérations au cours des entretiens. Toutes les séances étaient enregistrées en vidéo, le cadrage portant sur l'espace de travail et permettant de saisir les gestes du compositeur (en zoomant lorsque nécessaire). L'ensemble des données ainsi produites a été systématiquement transcrit selon des conventions prenant en compte plusieurs caractéristiques du discours oral : respect des bribes, mention des pauses dans le discours, indication entre crochets des gestes et de leur référence (du type : « [main droite désigne brouillon «I », première ligne, puis mesure 67, flûte + violon, manuscrit] »). L'ensemble de ces trois étapes formait ce que nous avons appelé une remise en situation de composition 22 - un type d'activité qui, pour Philippe Leroux, s'avérait heureusement distinct aussi bien de la fabrication de discours autorisés que du travail de composition en tant que tel.

\section{Bienfaits et limites du dispositif de remise en situation}

Quelques particularités de ce dispositif de recueil de données génétiques/cognitives le distinguent des cadres plus habituels de sollicitation de la parole d'un créateur sur son art. Il replace les inscriptions constituant le dossier génétique dans leur relation avec l'activité qui, tout à la fois, les produit et s'appuie sur elles à tout moment : d'une situation de composition donnée, les anticipations (y compris les plus vagues) comme les possibles non explicités et non notés pourront être commentés par le compositeur, au même titre que les opérations d'écriture les plus manifestes et définitives. De même, par les règles et par la gradation des trois étapes mises en œuvre, le dispositif tend à court-circuiter la téléologie caractérisant a priori le discours d'un créateur sur son œuvre passée : lorsqu'un moment de composition est remémoré et reformulé dans sa complexité, ses implications décelables dans la partition apparaissent largement décorrélées des significations qui seront les siennes une fois le passage, le mouvement, l'œuvre achevés. En plaçant compositeur et chercheurs non pas dans un face-à-face, mais tous ensemble face au dossier génétique, ce dispositif transforme la relation habituelle entre le créateur et son intervieweur dans le sens d'une

22. Voir Nicolas Donin et Jacques Theureau, « Theoretical and Methodological Issues Related to Long Term Creative Cognition: the Case of Musical Composition », Cognition Technology \& Work, vol. IX, n 4, 2007, p. 233-251. Un exemple de transcription complète d'une séance est accessible dans la partie « documents annexes » du DVD-Rom De Voi(rex) à Apocalypsis, fragments d'une genèse. Exploration multimédia du travail de composition de Philippe Leroux, joint à L'Inouï, revue de l'Ircam, n $2,2006$. 
collaboration entre individus autonomes, guidée par des intérêts réciproques ${ }^{23}$. Le créateur est alors un informateur privilégié sur sa propre activité, auprès d'un collectif dont il est lui aussi membre en tant qu'il prend part au dispositif de recherche24. Ainsi, contrairement à ce que l'emploi de la vidéo pourrait laisser superficiellement supposer, ces séances ne visaient pas à assouvir les pulsions scopiques éventuelles des chercheurs : non seulement elles avaient lieu à environ un an et demi de distance par rapport aux périodes d'écriture étudiées, mais leur enregistrement audiovisuel, loin de prendre part à une esthétisation de l'acte créateur, servait de documentation transitoire entre le moment de la séance et sa translation dans une transcription textuelle qui en conserve toute l'information utile (à l'exception toutefois de certaines données sonores/musicales, telles que des fichiers joués par le compositeur sur son ordinateur au cours de la séance, et qui sont donc référencés dans les transcriptions par leur minutage vidéo, facilitant leur consultation ultérieure).

Des produits de ce dispositif, différents usages sont concevables selon que l'on met l'accent sur le processus compositionnel ou sur son résultat, sur l'économie interne des documents génétiques ou sur celle de l'activité qui les a mobilisés, sur les déterminants sociohistoriques du projet artistique ou sur ses effets d'émergence les moins assignables à des règles ou des raisons. En l'occurrence, les transcriptions d'entretiens ont été segmentées en autant de fragments que d'opérations de composition explicitées (telles que : "Choix de voix pointilliste en relation de contraste avec le 1er mouvement », "Structure d'anticipation pour les quatre premières lettres du poème », « Relation entre instrumentation et accords aux mesures 91-92 »...). Ces fragments ont été réordonnés en une chronique aussi continue que possible, documentant la rédaction de Voi(rex) de la première à la dernière mesure. La nomination des fragments a permis de mettre en évidence des thèmes d'activité récurrents et d'identifier de façon synthétique les matériaux et procédures en jeu à chaque fois. Les analyses pouvaient par conséquent s'appuyer sur des réductions de la chronique suivant tel(s) ou tel(s) thème(s) d'activité, sans perdre de vue la continuité et la globalité du cours d'action dont ces moments et logiques étaient abstraits. Un premier pas vers l'analyse avait été réalisé au cours des séances avec le compositeur, puisque s'y était élaborée une réflexivité à propos de l'activité qui serait ensuite objet d'analyse. Mais ce caractère pré-analytique des données recueillies ne préjuge pas de la variété des modes d'analyse praticables sur l'ensemble formé du dossier génétique et des transcriptions des séances. Le présent article n'est pas le lieu de restituer des échantillons représentatifs de ces analyses, déjà extensivement publiées

23. Ici, l'intérêt explicite des chercheurs était l'accès à des informations riches et systématiques sur la composition d'une œuvre, tandis que l'intérêt explicite du compositeur était à la fois de connaissance de soi et de contribution à un projet collectif destiné à donner de l'intelligibilité à la musique contemporaine, sous plusieurs formes (articles scientifiques mais aussi réalisations multimédia), à travers l'une de ses propres œuvres.

24. ... Avec les contraintes déontologiques qui accompagnent ce type de recueil de données : au cours de l'entretien, le compositeur pouvait signaler qu'il souhaitait garder confidentiel tel ou tel propos qu'il venait de tenir, c'est-à-dire que la transcription de ce propos pourrait être utilisée dans l'analyse, mais non donner lieu à publication (cette clause a été mobilisée au plus une demi-douzaine de fois dans l'ensemble des entretiens sur Voi(rex) et Apocalypsis). De même, nous nous engagions à soumettre nos publications au compositeur pour validation : il pouvait formuler des réserves sur nos résultats et demander des modifications de nos transcriptions de sa parole. À l'usage, cette clause n'a pas mené à l'altération de nos conclusions ni de nos transcriptions, mais a principalement contribué à l'identification de faux-sens et d'ambiguïtés non résolues dans la reconstruction. 
ailleurs 25 . Mentionnons seulement quelques exemples des thèmes abordés : dynamique de la concrétisation d' «idées sonores » (notée sous forme graphique et/ou verbale) en relation avec différentes situations de composition ; mise en temps de structures précomposées et de plans, ajustés, révisés voire abandonnés au cours de leur implémentation rédactionnelle ; implications du milieu technologique, notamment des interfaces de logiciels, sur l'invention musicale ; incidences de la pratique de relecture/réécoute intérieure au fil de l'écriture ; individuation réciproque de l'œuvre en projet et de l'atelier en chantier ; co-construction de l'œuvre et du discours d'accompagnement de l'œuvre (préface à la partition, notice du programme de concert).

Ce projet de recherche était complété par deux autres projets, non moins expérimentaux, menés en parallèle : l'un, technologique, consistait (pour l'équipe Analyse des pratiques musicales en collaboration avec Philippe Leroux) à réaliser des prototypes de dispositifs multimédia d'exploration de Voi(rex) suivant des traits pertinents d'écoute selon le compositeur ; l'autre, artistique, consistait (pour Leroux) à engager la composition d'une nouvelle œuvre qui remobiliserait des idées, matériaux, techniques de Voi(rex) ainsi que des aspects de son processus de création dont la mémoire était alors entretenue par les séances de remise en situation de composition. Ces projets sont présentés et discutés ailleurs ${ }^{26}$, mais il convient de faire ici mention d'une réalisation à mi-chemin entre l'étude que l'on vient de présenter et le projet technologique : l'outil de «navigation génétique » dans Voi(rex) développé par plusieurs collaborateurs sous la direction de Samuel Goldszmidt. Cette base de données permettait l'intégration de l'ensemble des documents de genèse ainsi que des fragments d'entretien mis en chronique, et leur consultation au sein d'une interface ordonnant temporellement tous ces documents autour de la partition manuscrite associée à l'enregistrement de l'œuvre. Les extraits de la «chronique » permettaient d'assigner avec précision des parties du dossier génétique à des portions de la partition; le cours de cette dernière étant approximativement homologue au cours de sa rédaction, un parcours linéaire du manuscrit (enrichi par l'écoute de l'enregistrement) donnait accès, en accéléré, à l'enchaînement des principales opérations d'écriture et de pensée documentées par l'étude27.

\section{Vers la simultanéité entre genèse et étude génétique}

La collaboration précédente ayant été jugée concluante par tous ses participants, un dispositif analogue a été appliqué à une autre œuvre de Leroux, Apocalypsis, mais cette fois au cours même de sa composition. De septembre 2004 à mai 2006, Jacques Theureau et moi avons procédé avec le compositeur, à intervalles réguliers d'environ un mois et demi,

25. Une présentation générale du projet et une bibliographie in progress sont réunies dans <http://apm.ircam.fr/ leroux/>.

26. Pour le volet artistique, on pourra se reporter au texte de Philippe Leroux, ainsi qu'aux références qu'il mobilise, dans le présent volume. Pour le volet de médiation technologique, on pourra se reporter à Nicolas Donin, « Pour une "écoute informée" de la musique contemporaine : quelques travaux récents », Circuit, Musiques contemporaines, vol. XVI, n³, 2006, p. 51-64.

27. Une description de la «navigation génétique », précédée par une introduction générale à l'étude sur Voi(rex) comprenant plusieurs résumés d'analyses, a récemment paru : Nicolas Donin, « Genetic Criticism and Cognitive Anthropology: A Reconstruction of Philippe Leroux's Compositional Process for Voi(rex) », dans Genetic Criticism and the Creative Process: Essays from Music, Literature, and Theater, dir. William Kinderman et Joseph E. Jones, Rochester, University of Rochester Press, 2009, p. 192-215. 
à une remise en situation pour chaque jour de travail écoulé depuis la séance précédente. Cette génétique in vivo, ou plus exactement « en léger différé », s'est appuyée, d'une part sur l'état courant des différents fichiers (sons, routines informatiques, etc.), notes, esquisses, brouillons, et débuts d'écriture de la partition d'Apocalypsis, d'autre part sur un agenda de composition (un journal de bord succinct sous forme de liste de « choses faites ») rempli quotidiennement par le compositeur, à notre demande. Cette intervention - limitée mais réelle - dans l'écosystème compositionnel semblait la seule façon d'éviter un biais rétrospectif (interpréter toute l'activité des semaines passées à la lumière de la détermination actuelle de l'œuvre) qui, dans le cas de Voi(rex), avait pu être minimisé, d'abord grâce aux aide-mémoire portés dans la marge infrapaginale par le compositeur au fil de l'écriture, ensuite du fait que ce dernier n'était plus engagé dans la production de cette œuvre au moment du projet de recherche. Disposer d'un garde-fou fiable était d'autant plus utile que le projet d'Apocalypsis avait été conçu par le compositeur, comme on l'a dit, en rapport étroit avec Voi(rex) : la mise en abyme d'une œuvre par la suivante aurait pu facilement se transformer en dispositif de déformation téléologique permanente... Enfin, le recueil des données s'est adapté à cette nouvelle situation : puisque les séances recouraient à un ensemble de documents dont l'aspect évoluait continûment au cours du processus créateur, chaque séance s'accompagnait d'une duplication (photocopiage) de la totalité du dossier génétique dans son état actuel ; en outre, l'abondance des points à traiter (une à six opérations de composition pour chaque entrée du journal) a induit un dédoublement des séances : une première passe (généralement le matin) consistait à parcourir, et expliciter par des paraphrases, l'intégralité des entrées du journal couvrant la période considérée ; la seconde (généralement en fin d'après-midi) était un retour détaillé sur certains moments ou problèmes de composition, sélectionnés par les chercheurs à l'issue du parcours du matin.

Par rapport à l'étude précédente, un tel suivi de l'activité de composition a permis d'aborder systématiquement, outre la période d'écriture de la partition manuscrite, les périodes qui la précèdent (élaboration du projet et des matériaux) et la suivent (corrections de la partition, ajustements de dernière minute) - périodes qui, dans le cas de Voi(rex), n'avaient été abordées qu'indirectement. Le grain d'analyse est aussi incomparablement plus fin : la précision est de l'ordre du jour pour Apocalypsis vs une précision de l'ordre de la semaine ou du mois pour Voi(rex). Des phénomènes jusqu'alors invisibles sont apparus. Ainsi, quatorze séances ayant généré quatorze états intermédiaires du même dossier génétique, il devenait possible d'interroger en détail les logiques de remplissement progressif des brouillons grâce à la mise en série de ces derniers, ou encore d'émettre des hypothèses sur les relations entre documents de genèse et état du manuscrit au moment précis de saisie du travail en cours dont la séance avait fourni l'occasion. La figure 4 montre une page de la partition en cours de rédaction, telle qu'elle pouvait être vue le 11 octobre 2005 lors de la séance de remise en situation de composition couvrant la période du 8 septembre au 10 octobre (voir fig. 5). Jusqu'à ce jour, nous savions que Leroux esquissait la plupart des passages à même le manuscrit (c'est l'une des raisons pour lesquelles il écrit toujours au crayon, des esquisses aux brouillons et à la partition définitive) ; nous ignorions, en revanche, qu'il pouvait aussi porter des inscriptions anticipatoires une, deux ou plusieurs mesures en avance. L'annotation schématique placée dans la partie droite de la marge supérieure s'avéra en quelque sorte le chaînon manquant entre les brouillons formels (ou «feuilles d'idées » dans la terminologie du compositeur) 
sur lesquels est déterminé le contenu séquentiel d'une section ou d'un mouvement, et le travail rédactionnel correspondant sur le manuscrit (esquisses puis validation). Ce schéma est plus précis que ceux prescrivant, au sein des brouillons, le même passage ; il est l'ultime étape encore iconique et verbale avant la littéralisation solfégique qui figera bientôt l'idée sous forme de texte musical, avec des notes, des rythmes, une nuance et une articulation déterminés. Alors que cette dernière anticipation porte sur plusieurs mesures, les aidemémoire inscrits au milieu des portées (voir fig. 4 au centre de l'image) ont une portée

Fig. 4 : Manuscrit d'Apocalypsis, p. 25, photocopié lors de la séance de remise en situation de composition du 11 octobre 2005

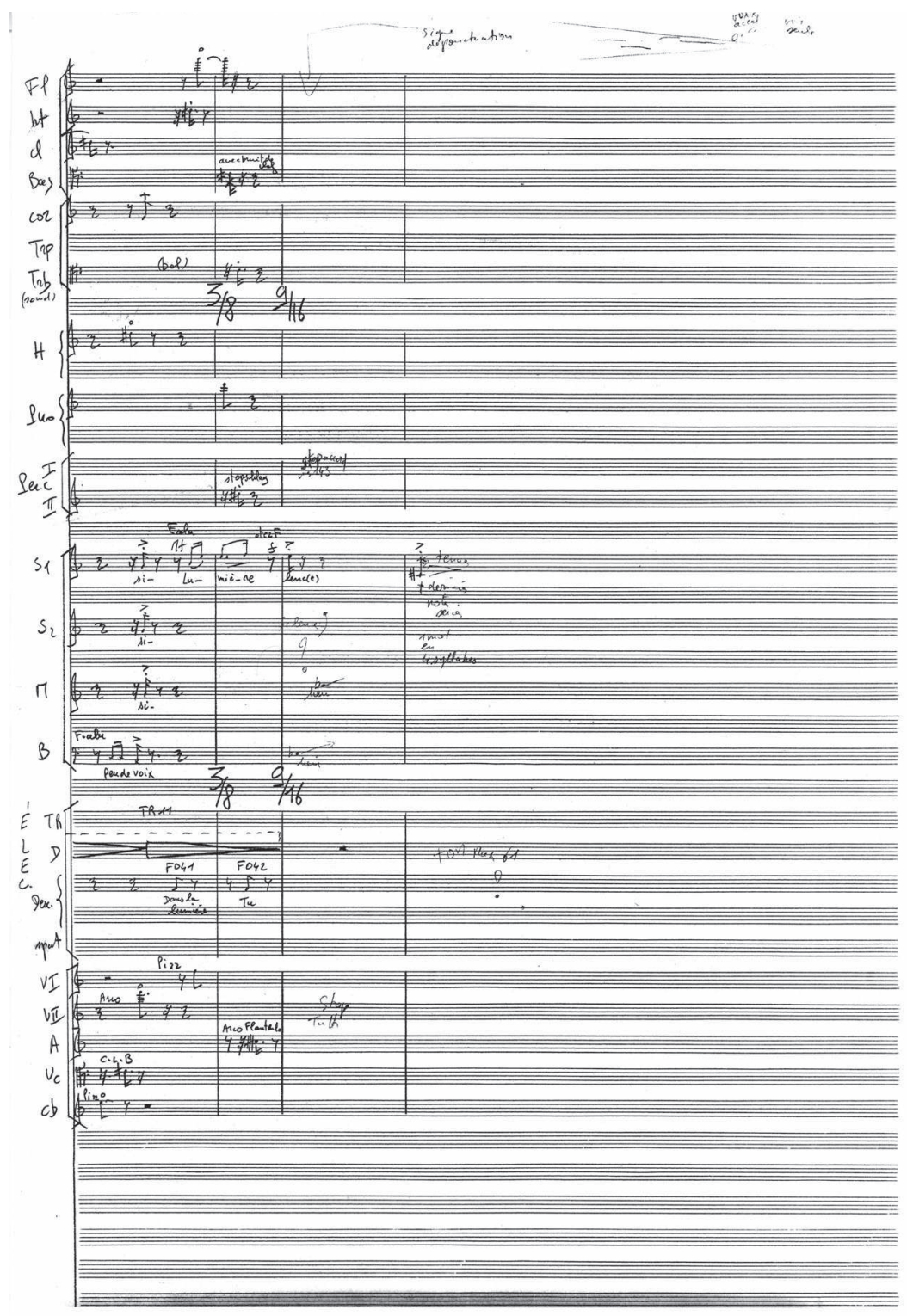




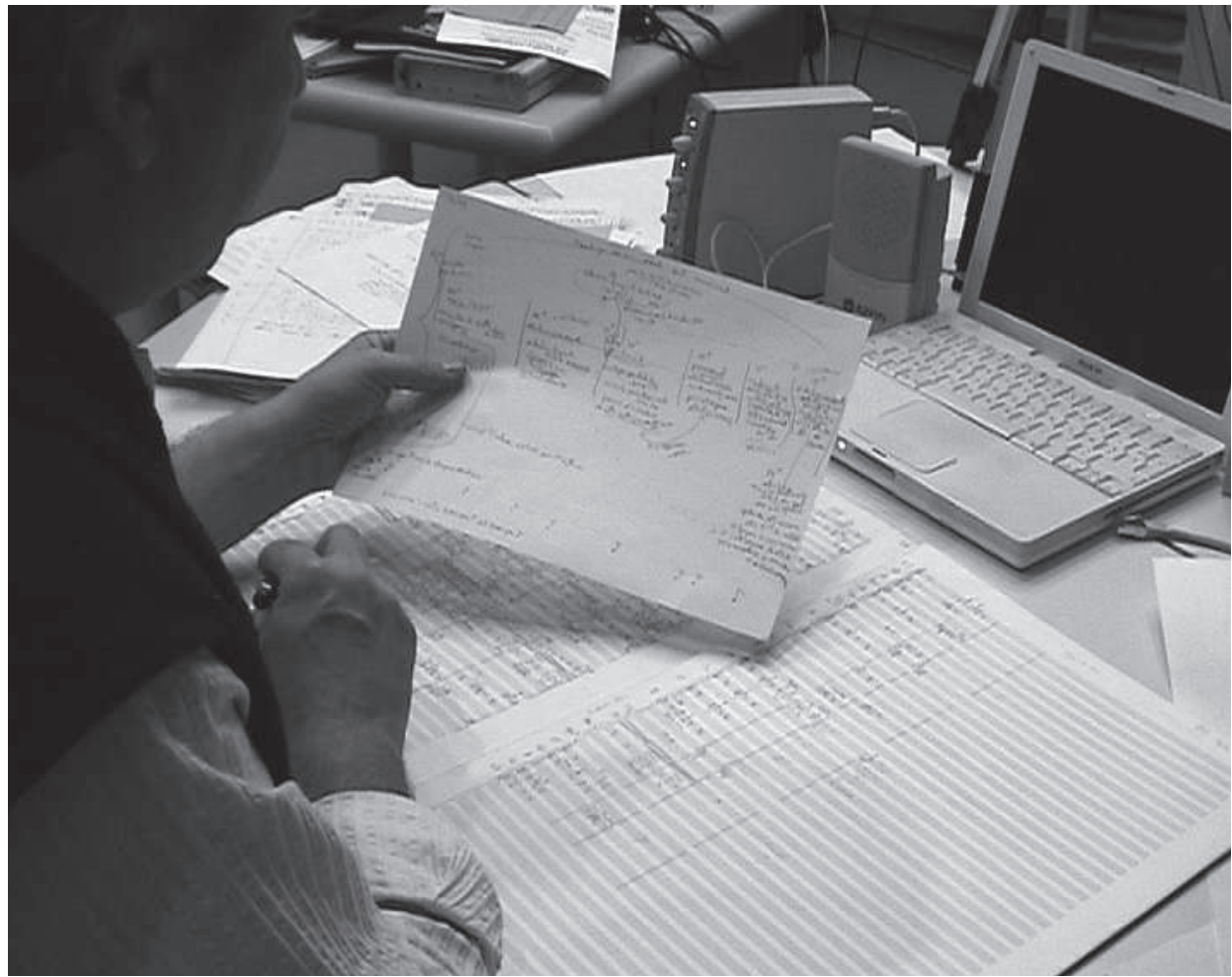

plus locale (ils commentent ce qui sera inscrit à leur emplacement) et plus immédiate (ils doivent servir à faciliter la reprise du travail après une interruption, en facilitant le rappel des critères de pertinence et des alternatives en jeu dans la rédaction des deux mesures en cours d'écriture). Ce n'est qu'à la faveur de l'étude in vivo que ces diverses traces, systématiquement effacées au fil de l'écriture, pouvaient finir par apparaître. Une fois leur existence et leur importance établies, nous avons demandé au compositeur d'éviter de gommer, autant qu'il lui était possible, les annotations marginales de ce type afin qu'elles puissent faire l'objet de commentaires au cours des séances de remise en situation de composition restantes, au même titre que les autres traces significatives du processus créateur. Comprendre la logique de reformulation des idées entre le brouillon et les marges du manuscrit n'était pas seulement une nécessité, du reste, pour la seule analyse génétique de cette œuvre particulière. Ainsi les études beethovéniennes, riches de quelque huit mille pages d'esquisses, ont mis en évidence l'existence de procédures comparables chez Beethoven (notamment l'inscription marginale d'un synopsis d'idées à l'aplomb de barres de mesures tracées en avance) mais n'ont pu les documenter que dans de très rares cas d'interruptions brutales de l'écriture 28 . Analyser la façon dont un compositeur d'aujourd'hui exploite les propriétés matérielles d'un support d'écriture qu'il partage avec de nombreux compositeurs du passé devrait permettre, en retour, d'enrichir les questions que l'on pose aux archives du travail créateur de ces derniers.
Fig. 5 : Philippe Leroux commentant la page 25 d'Apocalypsis en cours d'écriture, au moyen d'un brouillon séquentiel du mouvement III, pendant la séance de remise en situation de composition du 11 octobre 2005

28. Barry Cooper, Beethoven and the Creative Process, Oxford, Clarendon Press, 1990. 
Le caractère, sinon longitudinal, du moins prolongé, de notre collaboration avec Philippe Leroux a permis d'éclairer réciproquement la genèse de Voi(rex) et celle d'Apocalypsis : l'une et l'autre partageaient des traits spécifiques mais laissaient aussi voir la variabilité d'implémentation des procédures et notions qu'elles reconduisaient, ce qui permettait d'émettre des hypothèses sur la généralité de certaines caractéristiques observées (à plusieurs niveaux : pour Leroux, pour tout compositeur de musique contemporaine, pour tout compositeur de musique occidentale savante, etc.). En revanche, certains aspects de la pratique musicale considérée étaient moins bien documentés du fait du dispositif adopté. C'était particulièrement le cas de la dimension collaborative de la composition (soit, en l'occurrence, les interactions avec des interprètes et des réalisateurs en informatique musicale). Cette extension du travail créateur à d'autres mains que celle de l'auteur principal n'est pas forcément déterminante dans n'importe quel processus compositionnel, mais elle a eu historiquement une grande importance dans le développement de la musique contemporaine depuis l'après-Seconde Guerre mondiale, du fait de l'association étroite entre compositeurs d'avant-garde et lieux d'invention technico-scientifique (studios de musique électroacoustique, centre de recherche universitaires en informatique, collectifs d'expérimentation à la frontière art/science). Les projets de « recherche musicale » développés dans quelques-uns de ces lieux (dont l'Ircam tout au long de son existence) ${ }^{29}$ constituent des observatoires privilégiés de l'interaction entre compositeurs et les autres acteurs de l'innovation technologique et musicale. Afin d'explorer cette interaction, nous avons récemment mené une étude in vivo d'un projet de ce type impliquant plusieurs membres de l'Ircam et plusieurs instrumentistes réunis par la compositrice Florence Baschet (née en 1955) au long de deux années.

Élargissement du cadre d'observation : suivi d'un travail créateur collectif
Rendre compte du processus créateur supposait ici de documenter non seulement le travail de la compositrice, mais toutes les interactions engageant l'œuvre à venir au sein d'un collectif dont - surcroît de difficulté - une partie des participants se trouvaient être des collègues des musicologues-généticiens-ethnographes. L'objet de leur recherche musicale était un «quatuor augmenté » (vocable créé pour l'occasion), soit une œuvre musicale comprenant une partie électronique en temps réel et destinée à un quatuor à cordes dont chaque archet/poignet a été préalablement « augmenté »de capteurs de mouvement (capteur de pression sur la mèche de l'archet et gyroscope au poignet), permettant une interaction entre son instrumental et son électroacoustique en partie réglée par les gestes des musiciens. Le plan de travail défini par la compositrice en collaboration avec la direction artistique de l'Ircam (courant 2006) avait déterminé les modalités du travail collectif autour de sessions d'expérimentation avec les musiciens du Quatuor Danel, auquel l'œuvre était destinée : pendant la première année (septembre 2006-mai 2007), développement informatique et technique du dispositif de captation du geste instrumental par des membres de l'équipe de recherche Interactions musicales temps réel (Ircam-CNRS), et expérimentations avec chaque instrument seul puis avec plusieurs instrumentistes (soit

29. Pour une définition de la « recherche musicale » basée sur une enquête socio-ethnographique, voir Pierre-Michel Menger, Les Laboratoires de la création musicale : Acteurs, organisations et politique de la recherche musicale, Paris, La Documentation française, 1989. 
en duo soit en quatuor), à raison d'une session de trois heures tous les mois et demi en moyenne (voir fig. 6) ; pendant la seconde année (septembre 2007-printemps 2008), période de production essentiellement centrée sur le travail en studio de la compositrice et du réalisateur en informatique musicale qui l'assiste, Serge Lemouton. L'achèvement du projet s'est concrétisé de plusieurs façons : une œuvre musicale pour quatuor et électronique, StreicherKreis, a été composée (essentiellement pendant l'été 2007) et donnée en création (Ircam, novembre 2008) ; plusieurs développements informatiques spécifiques ont été réalisés et validés ; de nouveaux savoir-faire ont été éprouvés, des méthodes expérimentées. Notre étude consistait à documenter les formes, pratiques et techniques musicales qui s'inventent dans l'interaction entre plusieurs acteurs, aux compétences multiples et différenciées, d'un tel projet. Moteur et finalité du processus, la composition d'une œuvre par Florence Baschet constituait alors un cas d'étude privilégié pour aborder la complexité d'une genèse artistico-technologique. En outre, l'importance donnée au travail des interprètes (l'objectif explicite du dispositif de captation était de leur rendre le contrôle de détails du traitement du son en temps réel, d'habitude entièrement dévolu au compositeur et aux ingénieurs du son) garantissait un débordement de la génétique compositionnelle sur une génétique de l'interprétation.

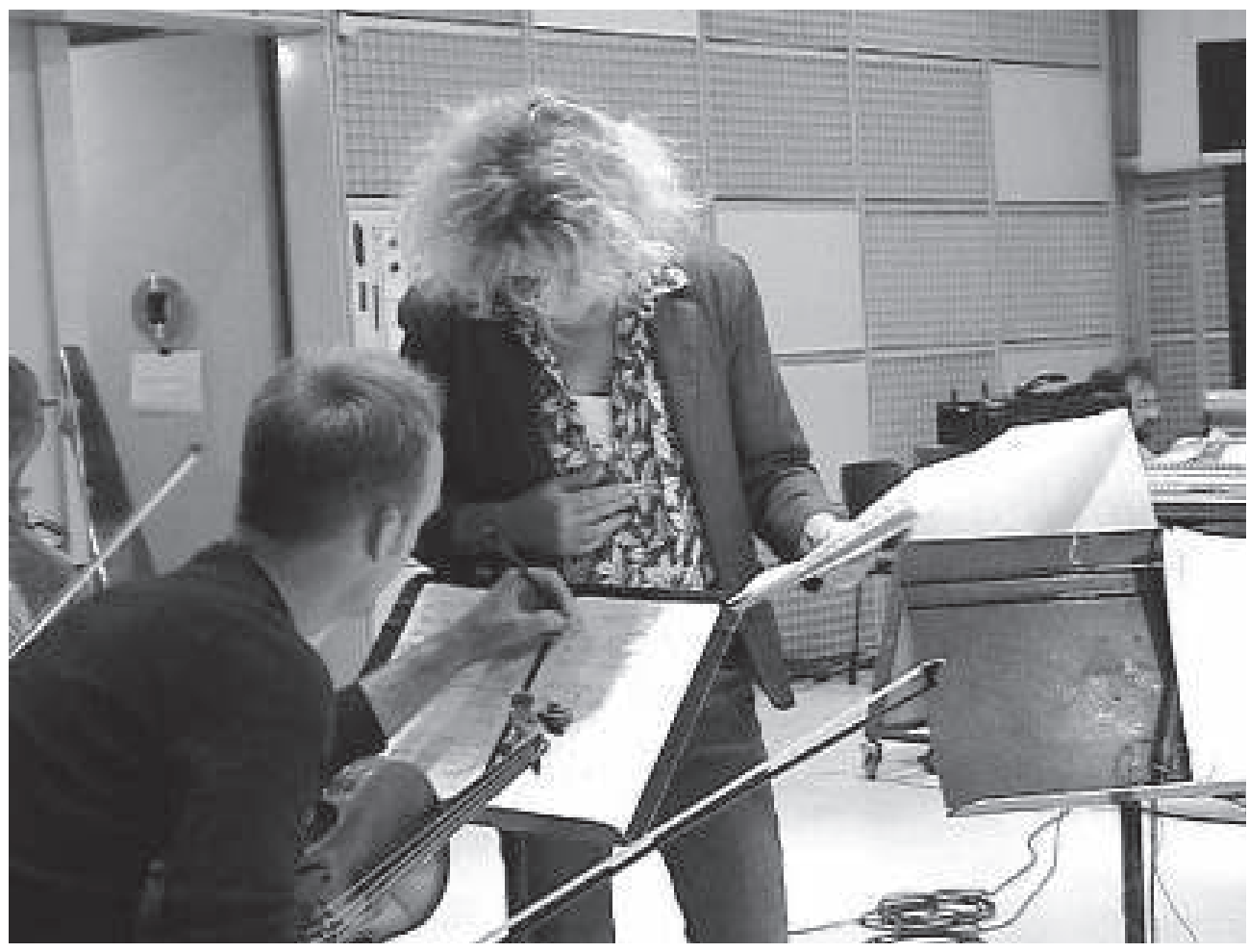

Fig. 6: Florence Baschet et l'un des musiciens du Quatuor Danel réglant ensemble l'exécution d'un passage de la partition au cours d'une séance de travail collectif à l'Ircam (printemps 2008) 
Plusieurs approches simultanées permettaient de saisir les différents points de vue engagés dans cette production collective : enregistrement vidéo des séances de travail (caméras mobiles permettant de retracer à la fois l'activité des instrumentistes et celle de l'équipe technique) accompagné d'une prise de notes ; enregistrement vidéo d'entretiens avec la compositrice, l'équipe de recherche et développement et le réalisateur en informatique musicale, juste après les séances afin d'en faire le bilan et en dégager les perspectives de travail pour ces derniers, juste avant les séances afin de faire le point sur le travail accompli depuis la séance précédente et de préciser ce qu'ils attendaient de la séance en question ; photocopie des fragments de partition utilisés ; sauvegarde des données informatiques produites au cours de la séance ; copie des rapports sur la séance précédente rédigés par la compositrice pour chaque séance ; copie des principaux échanges de courrier électronique entre la compositrice et l'impresario du quatuor ; last but not least, des entretiens individuels avec la compositrice centrés sur l'autonomie de son activité de composition. En particulier, grâce aux entretiens rétrospectifs/prospectifs régulièrement menés avec la compositrice, le chercheur-développeur et le réalisateur en informatique musicale, on dispose d'un historique du processus de conception du «quatuor augmenté » permettant de voir comment le plan de travail initial a été (parfois) ajusté et modifié au fur et à mesure de l'expérience accumulée au cours des séances ; aussi et surtout, on peut retracer de quelle façon les enjeux compositionnels, technologiques/ scientifiques et d'exécution instrumentale se sont réciproquement influencés. Quelques exemples en sont discutés dans la première publication issue de cette recherche ${ }^{30}$; d'autres publications sont en préparation, incluant un site web permettant la navigation dans une sélection de données commentées et problématisée par des articles scientifiques.

L'objet de cette étude est spécifique : par l'identité des acteurs et par sa temporalité, il ne ressemble à aucun autre (y compris même la production d'un spectacle musical ou celle d'une installation sonore) - et cette spécificité pourrait paraître excessive. Il nous a semblé que, loin de la condamner a priori à n'avoir de sens et d'intérêt que localement, une telle spécificité plaçait notre entreprise à la croisée de plusieurs perspectives, constitutives de facto d'une génétique élargie à un objet plus composite que la seule production d'un texte musical par Florence Baschet. Quand se substitue au jeu de quatuor normal un jeu de quatuor perturbé (où le retour auditif de chaque instrumentiste autant que les réglages interactionnels entre tous sont altérés par le traitement du son contrôlé en temps réel par leurs propres gestes), la génétique de l'interprétation ${ }^{31}$ gagne non seulement un cas exotique mais aussi un regard critique, une instructive négativité, sur tout ce que le jeu «normal » a si bien naturalisé qu'il en est devenu inaccessible à l'observation. Quand un projet créateur cible explicitement l' ' interprétation » en en faisant à la fois un enjeu technologique et un paramètre de l'écriture même de la partition, la génétique de la composition s'attaque à des thèmes d'activité essentiels mais habituellement invisibles, tant leur intégration au projet de l'œuvre se passe généralement de tout commentaire ou travail d'esquisse spécifique ; ces thèmes sont, par

30. «Organiser l'invention technologique et artistique ? L'activité collective de conception conjointe d'une œuvre et d'un dispositif informatique pour quatuor à cordes », Activités, vol. VI, n² 2, 2009, p. 24-43, téléchargeable à $<$ www.activites.org/v6n2/donin.pdf > ; voir également <http://apm.ircam.fr/quatuor/>.

31. ...ou de la «performance » (au sens anglais), si l'on veut bien subsumer sous cette catégorie aussi bien l'exécution musicale que le fait théâtral et plus généralement scénique. 
exemple : la façon dont le compositeur conçoit l'écriture instrumentale de sa future œuvre, la façon dont il prend en compte des particularités individuelles de ses premiers interprètes (généralement connus dès le moment de la commande de l'œuvre), la façon, enfin, dont il dose tout au long de l'écriture l'espace de liberté que le détail plus ou moins déterminé de sa notation laissera à tous ceux qui l'exécuteront. En outre, l'importance du travail sur la partie électronique de StreicherKreis, étudié à la faveur des périodes de collaboration en studio entre compositrice et réalisateur en informatique musicale, rappelle de nombreux projets compositionnels dans lesquels l'environnement intellectuel et technique d'une institution joue un rôle déterminant : en l'occurrence, la compositrice a décidé de renoncer aux patches informatiques avec lesquels elle travaillait depuis de longues années, pour reconstruire de zéro, avec l'aide de Serge Lemouton, de nouvelles versions actualisées de ces outils ; cette remise à plat d'une partie de l'atelier fut aussi l'occasion d'une prise de conscience de caractéristiques sensibles (rendu sonore, interface) des anciens patches qui ne pouvaient apparaître que par différence avec leurs successeurs. Enfin, un dernier type d'élargissement de l'objet génétique a été amorcé à la périphérie de ce terrain, par la documentation des versions successives des programmes informatiques réalisés pour le projet : c'était ici un cas typique de processus d'invention technologique contraint par une situation d'usage expert, qui se trouvait documenté au sein de la dynamique collective étudiée.

Une dernière caractéristique remarquable peut encore être commentée : le rythme régulier des séquences de travail lors desquelles Baschet amenait du matériau nouveau destiné à sa future œuvre (puis, pendant la seconde année, issu de son œuvre fraîchement écrite) a eu pour conséquence, là encore, de grossir démesurément des aspects du processus créateur en conférant à certaines de ses étapes un caractère public au sein du projet collectif 32 . La contrainte technique à la fois forte et instable (la compositrice rédigeait à chaque fois un texte destiné à tester la version actuelle du dispositif de captation du mouvement mais censée en même temps paver la voie à la future version fonctionnelle de concert) forçait les principaux protagonistes à verbaliser des implicites du projet qui, autrement, seraient restés des préoccupations individuelles et auraient laissé pas ou peu de traces. Même en se limitant strictement aux traces du travail solitaire de la compositrice, le matériau textuel produit et itéré à travers ces séances livre des informations génétiques précieuses : par exemple, le simple arrangement d'une « esquisse » existante pour un autre instrument du quatuor ${ }^{33}$, tout en répondant à un impératif de proximité maximale avec son modèle, implique localement de légères distorsions qui engagent des microdécisions musicales ; la façon dont ces dernières sont tranchées révèle parfois des oppositions distinctives que le travail de composition en tant que tel ne permettrait plus de distinguer, noyées parmi beaucoup d'autres.

32. Les éléments communiqués au quatuor par la compositrice avant chaque session, sous forme de fichiers pdf contenant un fragment de partition saisi par traitement de texte musical, étaient suggestivement intitulés par elle «Esquisses », même s’ils présentaient un caractère extrêmement abouti (phrases complètes, avec un début et une fin, articulées les unes aux autres en un tout apparemment autonome).

33. Adapter pour alto ou pour violoncelle telle phrase musicale initialement écrite pour le violon suppose, pour que les informations captées sur le geste soient susceptibles de comparaison, une transposition des notes sur les emplacements relatifs correspondant sur le manche de ces instruments. 
Ralentir (?) : travaux
Plusieurs travaux en cours cherchent à articuler et à confronter des études génétiques in vivo et des études de format plus classique portant sur des œuvres et compositeurs du passé. Comme on l'a relevé supra, il y a fort à parier qu'une génétique des œuvres en train de se faire pourrait identifier, ou rouvrir, des problématiques intéressant la génétique en général.

Une première tentative, réalisée à l'occasion de la conception d'un numéro thématique de revue 34 , a consisté à mettre en série différents « ateliers » représentatifs de la musique contemporaine : d'une part ceux d'Elliott Carter, José Evangelista, Jonathan Harvey, Salvatore Sciarrino et d'un réalisateur en informatique musicale (Gilbert Nouno), saisis sur le vif à partir d'approches tantôt génétiques, tantôt ethnographiques, et, pour Harvey, sous la forme d'une introspection ; d'autre part, à travers des analyses plus complètes et thématisées : modalités citationnelles et autocitationnelles dans une œuvre de György Kurtág, intégration sélective de possibles technologiques au cours de la composition d'œuvres de plusieurs compositeurs à l'Ircam dans les années quatre-vingt et deux mille ; le dossier était complété par une enquête auprès d'une vingtaine de compositeurs nordaméricains sur leur emploi créatif et pédagogique de l'ordinateur. La réunion de cet ensemble de travaux faisait apparaître des traits partagés par plusieurs des démarches créatrices considérées : distinction entre travail paramétrique et «schémas » évocateurs de phénomènes sonores ; importance des pratiques de recyclage et d'arrangement au cours de l'écriture (indépendamment du positionnement esthétique du compositeur à l'égard du clivage moderne/postmoderne); lorsqu'il y a usage de l'informatique, fonctions différenciées des esquisses audionumériques selon les logiciels adoptés ; vigilance du compositeur à l'égard des connotations stylistiques pouvant apparaître sous sa plume (ou au fil de son utilisation de l'informatique musicale); caractère généralement heuristique et seulement partiellement prescriptif des plans formels préalables à la phase de composition en tant que telle.

Le projet « Musicologie des techniques de composition contemporaines 35 », mené par un groupe de dix chercheurs francophones de 2009 à 2011 et soutenu par des subventions de l'Agence nationale de la recherche (France) et de la Haute École spécialisée de Suisse occidentale, tente de reconduire cette approche à l'échelle d'un programme de travail coordonné dans lequel une demi-douzaine de processus créateurs, relevant tous de la «musique contemporaine » mais dépourvus de filiations ou points de comparaison explicites, sont explorés en parallèle. La plupart composent avec une contrainte pragmatique (généralement technique ou technologique), susceptible de servir d' « analyseur » du processus dans sa totalité, et l'ensemble est réparti sur toute la période historique de développement de la musique contemporaine - de la musique de Charles Koechlin pour le film Victoire de la vie de Cartier-Bresson à la conception d'une installation sonore pour un jardin public parisien par Jean-Luc Hervé (2008-...) en passant par l'opus fondateur de Marco Stroppa, Traiettoria pour piano et sons de synthèse (1982-

34. Circuit. Musiques contemporaines, « La fabrique des œuvres », dir. Nicolas Donin et Jacques Theureau, vol. XVIII, n $1,2008$.

35. Voir <http://apm.ircam.fr/mutec/>. 
1988) ${ }^{36}$. Chacun des dossiers abordés permet d'engager un dialogue avec la littérature scientifique existante sur des processus de création musicale comparables ou corrélés, mais aussi de croiser des thèmes d'analyse traversant la plupart des autres dossiers : étagement temporel de la prise en compte des contraintes techniques, rapport entre planification (et/ou programmation informatique) et réalisation, points de convergence et de divergence entre formulations verbales, graphiques, solfégiques du projet, etc.

L'une des études actuellement menées dans ce cadre est consacrée à la composition d'une nouvelle œuvre, Gramigna pour cymbalum et petit ensemble (2009-...), par Stefano Gervasoni (né en 1962). Ici la contrainte poétique est particulière : composer des miniatures qui s'ajouteront aux quatre premières (constitutives d'une version germinale de l'œuvre datant de l'automne 2009), afin d'aboutir à un cycle dont tous les éléments seraient des greffes ou reprises d'autres œuvres récentes ou en cours - ou encore, inversement, seraient les laboratoires d'œuvres en chantier. Le processus compositionnel se veut interstitiel comme les « herbes folles » désignées par le titre, le cycle se constituant à la marge des œuvres plus officielles et ambitieuses avec lesquelles il cohabite. Les caractéristiques originales du projet de Gervasoni rejaillissent sur les modalités de recueil de notre dossier génétique in vivo (en collaboration avec François-Xavier Féron). Les pièces sont écrites rapidement (un ou deux jours peuvent suffire), dans des circonstances plus ou moins impromptues et plus ou moins expérimentales. Le compositeur esquisse peu, voire pas du tout, en dehors du gommage à même la partition manuscrite ; en compensation de cette difficulté, le travail d'écriture est très peu dépendant du lieu physique de l'atelier où Gervasoni compose habituellement. Pour mener à bien une remise en situation de composition relative à un processus créateur de quelques heures à peine et laissant peu de traces visibles, deux procédés ont été expérimentés. Dans un cas où le moment de la composition était éloigné de plusieurs semaines, nous nous sommes inspiré de la méthode du sosie (notamment pratiquée en psycho-ergonomie), refaisant nous-même, un par un sur du papier à musique vierge, des gestes d'écriture plausibles menant au remplissement du manuscrit à l'identique, et le compositeur pouvait à chaque instant interrompre et commenter ces opérations en évaluant leur pertinence et en aiguillant la reconstitution (voir fig. 7) ; ce procédé, s'il a laissé nécessairement de vastes zones de flou quant au détail du cours d'action considéré, a permis de démêler de nombreuses implications cognitives sous-tendant la partition. Dans un autre cas, le compositeur s'est installé à l'improviste dans une salle attenante à notre bureau pendant l'un de ses fréquents séjours parisiens et a souhaité y composer plusieurs pages d'une nouvelle pièce. Nous avons eu son accord pour pratiquer de régulières mais silencieuses intrusions visant à documenter les étapes de l'écriture, sans l'interrompre, par la photographie de son espace de travail tous les quarts d'heure ; la série d'images ainsi produite a servi de guide à une remise en situation de composition quelques jours plus tard. Et ce ne sont certainement pas là les dernières reconfigurations méthodologiques nécessaires pour s'adapter aussi « écologiquement » que possible aux conditions évolutives dans lesquelles continue de s'écrire Gramigna...

36. Les premiers résultats de l'une de ces études, consacrée à la genèse des Espaces acoustiques de Grisey, sont présentés à travers le sous-ensemble du dossier génétique édité et commenté par François-Xavier Féron dans le présent numéro de Genesis. 
Fig. 7 : Stefano Gervasoni et Nicolas Donin pendant la séance de remise en situation de composition de Gramigna du 19 janvier 2010 (photogramme extrait d'un enregistrement vidéo) En haut à gauche : première page de la partition imprimée de Gramigna (éd. Suvini Zerboni, Milan).

En haut à droite : première page de la partition manuscrite. En bas à droite : copie (in progress) de la page de partition manuscrite, effectuée par le chercheur pour les besoins de la reconstitution

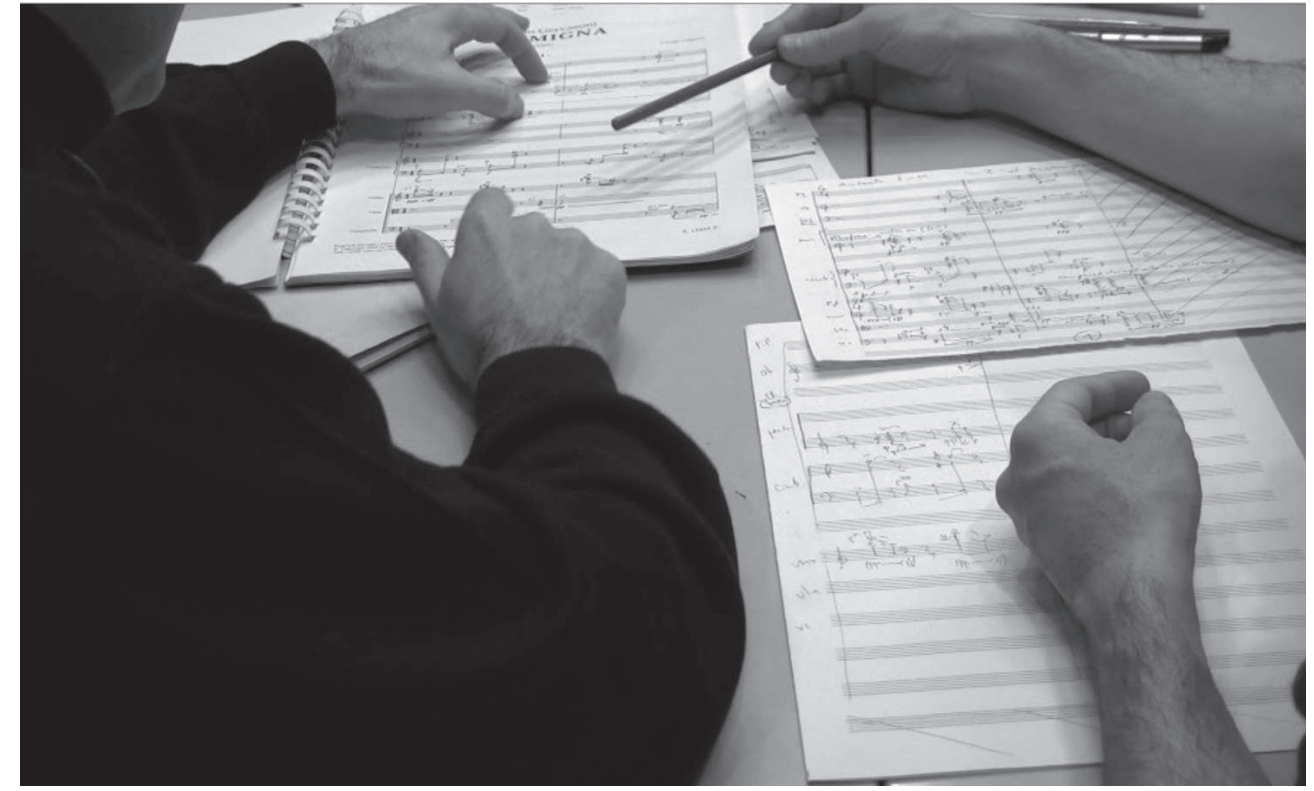

$* * *$

La génétique in vivo apparaît, au terme de ce parcours, comme la co-construction par le(s) chercheur(s) et le(s) créateur(s) d'un objet de recherche et de son matériau. Il faut s'entendre sur le sens positif à donner à cet énoncé. Qu'il y ait une nécessaire implication de la part du (des) créateurs(s) ne signifie pas que ce type de démarche génétique soit condamné à un défaut d'objectivité dont l'« autre » génétique ne serait jamais affectée ; on pourrait même s'amuser à soutenir le contraire en remarquant que le travail sur un corpus génétique post mortem ne permet pas souvent l'évaluation rigoureuse de la représentativité et des lacunes du dossier, pourtant nécessaires à son bon usage - ou encore, que l'impossibilité d'interagir avec les auteurs du passé nous prive de bien des occasions de démystifier dialogiquement les reconstructions involontaires (et les véritables fausses pistes) dont ils ont nourri les documents de travail qu'ils abandonnaient à la postérité. Une fois admis le caractère inévitablement construit et interactif de nos objets, il s'agit d'abord de limiter et de contrôler autant que possible les éléments artificiellement introduits par l'apparition d'une démarche de documentation et de recherche dans l'environnement du processus créateur considéré, puis de tenir compte, à toutes les étapes ultérieures d'analyse, des propriétés particulières du dispositif choisi.

Au vu de la relative diversité des dispositifs de recherche présentés dans cet article, il ne s'agit pas, on l'aura compris, de proposer une méthodologie rigide qui se voudrait universellement valable pour ce type d'études. Nous avons adopté une logique à la Lampedusa : pour qu'un même type d'objet soit à chaque fois saisi, il fallait (et il faudra) changer ou adapter les modalités de participation des chercheurs et de recueil des données en fonction des spécificités des pratiques et des situations étudiées. En revanche, insistons sur 
l'une des grandes difficultés à résoudre, celle-là même qu'une critique du documentaire de Clouzot nous avait permis d'introduire allégoriquement : théorie et méthode doivent être en cohérence l'une avec l'autre, et leur articulation doit être aussi explicite que possible dès la mise en œuvre du projet. On l'a vu au fil de cet article, dans notre conception de la génétique in vivo, il n'est pas de questions purement pratiques et le bon Dieu est dans les détails : le contrat entre les participants, le contenu des séances, le choix du lieu, le positionnement de la caméra et les modalités de cadrage sont dépendants d'une épistémologie avant de l'être de contraintes matérielles.

Il convient aussi de relever ici la communauté de vue entre la «musicologie de terrain» mise en œuvre dans nos travaux et le programme de la critique génétique. Tous deux partagent l'objectif de rendre compte des processus créateurs sur une base empiriquement fondée, donnent un caractère central aux esquisses, brouillons et manuscrits, recourent à une terminologie mutualisable (avant-texte, dossier génétique, écriture, etc.), et ont le souci de rendre compte à la fois de la singularité des effets esthétiques et de la généralité anthropologique. Si les démarches présentées dans cet article se laissent situer en partie à l'extérieur des frontières actuelles de la génétique, ce n'est donc pas du fait de leur exotisme disciplinaire, mais du faible développement de la critique génétique in vivo jusqu'à maintenant. Gageons que la défiance viscérale de la génétique à l'égard des mystiques en tous genres trouvera encore quelques forteresses à désarmer. 
Nicolas Donin est musicologue à l'Institut de Recherche et de Coordination Acoustique/Musique, où il dirige l'équipe Analyse des pratiques musicales (Ircam-CNRS). Il est l'auteur d'études sur la musique contemporaine, l'histoire des pratiques d'écoute et d'analyse musicale depuis la fin du XIXe siècle, et l'analyse musicale d'interprétation, publiées à la fois dans le champ musicologique et dans celui des sciences humaines. Il est aussi coauteur de courts-métrages, de DVD-Roms et de publications en ligne issus de ces recherches.

Nicolas Donin, Nicolas.Donin@ircam.fr

Résumés

\section{Quand l'étude génétique est contemporaine du processus de création}

L'idée d'une saisie du processus créateur sur le vif (pendant ou peu après son effectuation) a été formulée à plusieurs reprises, aussi bien par des artistes que par des chercheurs. Elle n'a que récemment donné lieu à une mise en pratique effective, inscrite dans une démarche scientifique explicite. L'article propose un compte rendu des différentes formes prises par cette « génétique in vivo » au fil de plusieurs études musicologiques réalisées par l'auteur en collaboration avec d'autres chercheurs et des musiciens : reconstruction de la genèse de Voi(rex) de Philippe Leroux avec la participation du compositeur ; toujours avec Leroux, documentation et analyse du processus de composition d'Apocalypsis au fil même du travail créateur ; suivi ethnographique de l'élaboration collective d'une œuvre et d'un dispositif technologique dans un projet de recherche musicale associant composition, interprétation, recherche et développement ; études croisées de processus créateurs passés et actuels dans le cadre d'un programme de recherche en cours.

The idea of capturing the creative process in action (during or shortly after its application) has been articulated repeatedly by artists as well as by researchers. It is only recently that it has actually been put into practice and inscribed in an explicit scientific process. This article presents a review of the various forms taken by these "live genetics", over successive musicological studies performed by the author with other researchers and musicians: reconstruction of the genesis of Voi(rex) by Philippe Leroux, with the participation of the composer; and again with Leroux, documentation and analysis of the composition process of Apocalypsis in the course of the creative work; ethnographic follow-up of the collective elaboration of a work and of a technological device in a musical research project associating composition, interpretation, research and development; cross studies of past and present creative processes within the framework of an ongoing research program.

Der Gedanke, den kreativen Prozess in vivo zu erfassen (während oder kurz nach seiner Ausführung), wurde immer wieder formuliert, sowohl von Künstlern als auch von Forschern. Erst in jüngerer Zeit wurde er in eine konkrete Praxis übersetzt, die sich durch eine eindeutig wissenschaftliche Vorgehensweise auszeichnet. Der Aufsatz bietet eine Übersicht über verschiedene Formen, in denen diese « Genetik in vivo » im Verlauf von mehreren musikwissenschaftlichen Studien des Verfassers mit anderen Wissenschaftlern und Musikern Gestalt angenommen hat : Die Rekonstruktion der Genese von Voi(rex) von Philippe Leroux unter Mitwirkung des Komponisten; ebenfalls mit Leroux, eine Dokumentation und Analyse der Komposition von Apocalypsis, im Prozess ihrer Entstehung; eine ethnographische Langzeitstudie über die kollektive Ausarbeitung eines Werkes sowie über eine technologische Struktur im Rahmen eines musikalischen Forschungsprojektes, in dem Komposition, Interpretation, Forschung und Entwicklung miteinander verknüpft sind; vergleichende Untersuchungen vergangener und gegenwärtiger Schaffensprozesse im Rahmen eines laufenden Forschungsprogrammes.
Tanto los artistas como los investigadores, han formulado en múltiples ocasiones la idea de una aprehensión del proceso creativo en el momento mismo de su realización o poco después de finalizada. Esta idea ha dado lugar recientemente a una puesta en práctica efectiva, inscrita en una experiencia científica explícita. Este artículo propone un panorama de las distintas formas adoptadas por esta "genética in vivo" a lo largo de varios estudios musicológicos realizados por el autor en colaboración con otros investigadores y músicos: reconstrucción de la génesis de Voi(rex) de Philippe Leroux con la participación del compositor; con el mismo Leroux, documentación y análisis del proceso de composición de Apocalypsis simultáneamente con el trabajo creativo; seguimiento etnográfico de la elaboración colectiva de una obra y de un dispositivo tecnológico en un proyecto de investigación musical que asocia composición, interpretación, investigación y desarrollo; estudios entrecruzados de procesos creativos pasados y actuales en el marco de un programa de investigación en curso.

A ideia de captar a quente o processo criativo (quando decorre ou logo depois) tem sido avançada várias vezes, tanto por artistas como por pesquisadores. Ainda recentemente inspirou uma experiência real, inscrita em explícito programa científico. Neste artigo são resenhadas diferentes formas assumidas por esta "genética in vivo" no decurso dos estudos musicológicos realizados pelo autor em colaboração com outros investigadores e músicos: reconstrução da génese de Voi(rex) de Philippe Leroux com participação do compositor; com o mesmo, documentário e análise do processo de composição de Apocalypsis a par do trabalho de criação; acompanhamento etnográfico da elaboração colectiva de uma obra e de um dispositivo tecnológico em projecto de pesquisa musical que associa composição, interpretação, investigação e desenvolvimento; estudos cruzados de processos criativos passados e actuais no âmbito de um programa de investigação em curso.

L'idea di afferrare il processo creativo in vivo (durante o poco dopo che sia stato effettuato) è stato formulato più volte, sia da parte degli artisti che degli studiosi. Solo recentemente è stato messo in pratica secondo un procedimento scientifico esplicito. L'articolo propone un resoconto delle diverse forme assunte da questa "genetica in vivo" sul filo di molteplici studi musicologici condotti dall'autore in collaborazione con altri studiosi e musicisti: ricostruzione di Voi(rex) di Philippe Leroux con la partecipazione del compositore; ancora con Leroux, documentazione e analisi del processo di composizione di Apocalypsis durante la fase stessa di creazione; osservazione etnografica dell'elaborazione collettiva di un'opera e di un dispositivo tecnologico nel progetto di ricerca musicale che associa composizione, interpretazione, ricerca e sviluppo; studi incrociati di processi creativi passati e attuali nel quadro di un programma di ricerca in corso 